\title{
Biolinguistic Diversity Index of India
}

\author{
Ritesh Kumar \\ Centre for Linguistics \\ Jawaharlal Nehru University \\ New Delhi, India \\ riteshkrjnu@gmail.com
}

\begin{abstract}
Harmon and Loh calculated the 'Index of Biocultural Diversity' (IBCD) at the globallevel (they calculated the index for each country and then compared them). However the problem with their methodology was that it could not be used for smaller areas or intracountry calculation of index. So it required some modification. In this paper I have calculated the IBLD (Index of Biolinguistic Diversity) of India using a similar methodology. However I have introduced some modifications. Instead of taking the politically divided states as the reference point for comparison, I have taken the ecoregions of India as the reference point. I have also calculated the Spearman's rank correlation coefficient between the ranks of the eco-regions on the basis of biodiversity and linguistic diversity so as to see whether these are correlated at the intra-country level (in India, in particular). The conclusions are not exactly at par with the expectations but still the correlation is established.
\end{abstract}

\section{Biocultural diversity: An Introduction}

Biocultural Diversity is a very recent concept — not more than a decade old - which is based on the fundamental assumption that there is an inextricable link between biological and cultural and linguistic diversity. It draws from anthropological, ethnobiological, ethnoecological, and linguistic insights regarding the relationships of human language, knowledge, culture and practices with the environment and nature.

Biocultural diversity unifies the diversity of life in all of its manifestations: biological, cultural, and linguistic. These are interrelated and have coevolved within a complex socio-ecological adaptive system. There are three basic assumptions underlying the concept of biocultural diversity.

1 The diversity of life does not only mean the diversity of plants and animal species, habitats, and ecosystems found on the planet, but also the diversity of human cultures and languages.

2 These diversities are not separate or exist in parallel realms, but rather they interact with and affect one another in complex ways.

3 The interaction among these diversities have developed over time through mutual adaptation between humans and the environment at the local level, which is, probably, of a coevolutionary nature and, thus, a causal one (Maffi, 2005, 2007a, 2007b).

The cumulative effect of all these local interlinkages, interdependencies and interaction between the humans and the environment implies that at the global level biodiversity and cultural diversity are also interlinked and interdependent. Thus, it has significant implications for the conservation of both the diversities. Recent global cross-mappings of the distributions of biodiversity and linguistic diversity (taken as a proxy for cultural diversity) have revealed significant geographic overlaps between the two diversities, especially in the tropics. Moreover, they have shown a strong coincidence between biologically and linguistically megadiverse countries (Loh \& Harman, 2005). 
The way in which the local and the global biocultural diversities interact is represented in the following diagram.

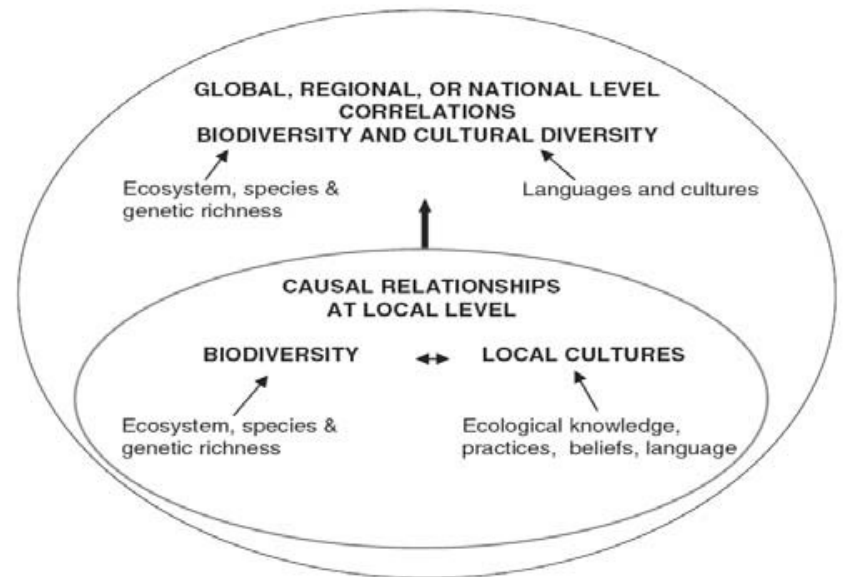

Fig1: Causal relationship at local level and its relation to the diversity at global level.

Source: original figure by Ellen Woodley, 2005

A global cross-mapping of the endemic languages and the endemic higher vertebrate species brings out the remarkable overlap between linguistic and biological diversity throughout the world. Similar results can be obtained by cross-mapping the endemic languages and the flowering plant species. Moreover, comparison of a list of endemic languages with the IUCN list of 'megadiversity' countries reveals that 10 out of the top 12 megadiversity countries (or 83\%) also figure among the top 25 countries for endemic languages (Skutnabb-Kangas, 2008).

It has been noted that generally the social factors combine with the geographic and climatic factors leading to a higher or lower diversity. For example, geography and climate of a particular area affects its carrying capacity and access to resources for human use. Ease of access to abundant resources seems to favor localized boundary formation and diversification of larger numbers of small human societies and languages. Where resources are scarce, the necessity to have access to a larger territory to meet subsistence needs favors smaller numbers of widely distributed populations and languages (Carder, 2008). The development of complex societies and large-scale economies, which tend to spread and expand beyond their borders, also correlates with a lowering of both linguistic and biological diversity. Moreover, there is a significant overlap between the location of threatened ecosystems and threatened languages. On the other hand, low population density, at least in tropical areas, seems to correlate positively with high biocultural diversity (Maffi, 1998).

\section{IBCD: An Index for Measuring Biocultural Diversity}

In order to sustain and protect biocultural diversity we must have a measure of the diversity we have; the diversity we are losing and the rate at which global diversity is decreasing. In 2005, Harmon and Loh developed the blueprint for an Index of Biocultural Diversity (IBCD), whose purpose is to measure the condition and trends in biocultural diversity on a country-to country basis (the level at which the available data sets are organized). Till then linguistic diversity alone was taken as the proxy for cultural diversity. But they took three cultural indicators: languages, ethnicities and religions as these were the aspects of culture for which global data (on a country-tocountry level) was available. Finally IBCD is calculated by aggregating data on the three cultural indicators with data on the diversity of bird/mammal species and plant species as indicators for biodiversity (also selected on the basis of global data availability).

The IBCD has three parts: 
-A biocultural diversity richness component (BCDRICH), which is a relative measure of a country's 'raw' biocultural diversity using unadjusted counts of the five indicators.

-An areal component (BCD-AREA), which adjusts the indicators for land area and therefore measures a country's biocultural diversity relative to its physical extent.

-A population component (BCD-POP), which adjusts the indicators for human population and therefore measures a country's biocultural diversity relative to its population size.

The IBCD gives equal weight to cultural and biological diversity, so a country's overall biocultural diversity score is calculated as the average of its cultural diversity score (CD) and its biological diversity score (BD).

$\mathrm{IBCD}=(\mathrm{CD}+\mathrm{BD}) / 2$

In measuring a country's cultural diversity $\mathrm{CD}$, equal weight is given to linguistic, religious and ethnic diversity. Therefore CD is calculated as the average of a country's language diversity (LD), religious diversity $(\mathrm{RD})$, and ethnic group diversity (ED):

$\mathrm{CD}=(\mathrm{LD}+\mathrm{RD}+\mathrm{ED}) / 3$

In measuring biodiversity $\mathrm{BD}$, equal weight is given to animal species diversity (using birds and mammals as a proxy for all animal species. Marine mammals are excluded from the analysis) and plant species diversity. Therefore BD is calculated as the average of a country's bird and mammal species diversity (MD), and plant species diversity (PD):

$\mathrm{BD}=(\mathrm{MD}+\mathrm{PD}) / 2$

Each indicator is given an equal Weightage as this is the simplest way of calculating the index. As an aggregated index, the IBCD could be calculated using different Weightage, to give greater or lesser importance to any of the five component indicators.

To derive country scores for each of the five component indicators, each country's richness value was compared with the global value. For example, for language diversity, LD is calculated as the log of the number of languages spoken in a country divided by the log of the number of languages spoken worldwide (see the Table above).

$\mathrm{LD}=(\log \mathrm{Li}) /(\log \mathrm{L}$ world $)$ where $\mathrm{Li}$ is the number of languages spoken in country $\mathrm{i}$, $\mathrm{L}$ world is the number of languages spoken in the world (currently 6800).

The calculation was repeated for the other four indicators to derive BCD-RICH.

To compensate for the fact that large countries tend to have a greater biological and cultural diversity than small ones simply because of their greater area (or greater population), two additional diversity values for each country was calculated by adjusting first for land area (BCD-AREA) and second for population size (BCD-POP). This was done by measuring how much more or less diverse a country is in comparison with an expected value based on its area or population alone.

The process was repeated for the other four indicators to derive BCD-AREA and BCD-POP. The expected diversity was calculated using the standard formula for the species-area relationship log $S$ $=\mathrm{c}+\mathrm{z} \log \mathrm{A}$, where

$\mathrm{S}=$ number of species,

$\mathrm{A}=$ area, and $\mathrm{c}$ and $\mathrm{z}$ are constants derived from observation.

To calculate the deviation of each country from its expected value, the expected log Ni value was subtracted from the observed log $\mathrm{Ni}$ value. The index is calibrated such that the world, or maximum, value is set equal to 1.0 , the minimum value is set equal to zero and the average or typical value is 0.5 (meaning no more or less diverse than expected given a country's area or population).

\section{Calculating the IBCD within the Ecoregions of India}


The first problem in calculating the IBCD within India was to decide upon the reference points within the country. At the global level it was the individual country that formed the reference point. I had two options before me-a) the politically demarcated 'states' of India, which has practically no scientific basis for their division; b) the eco-regions that the country is divided into by the WWF. Obviously, I chose the latter one as that is more scientifically divided on the basis of biological diversity. An eco-region is a relatively large unit of land that contains geographically distinct assemblage of natural communities with boundaries that approximate the original extent of natural communities prior to the major land use change. Eco-regions:

-share a large majority of their species and ecological dynamics;

- share similar environmental conditions, and;

-interact ecologically in ways that are critical for their long-term persistence. The whole globe is divided into 867 ecoregions and there are the organized data for the biological diversity in these regions.

For the purpose of calculating the IBCD of different ecoregions of India, I have adapted the methodology described above to suit my needs. And the index that I have calculated is not that of 'biocultural' diversity but rather it is something like 'biolinguistic' diversity. I have looked only at the correlation between linguistic diversity and biological diversity in these regions. So it might be rephrased as IBLD instead of IBCD. Moreover I have also not adjusted the data with reference to the area and population. So I have calculated just IBLD-RICH and no IBLD-POP and IBLDAREA. This has been done because of three reasons:

-My work is basically concerned with the linguistic and biological diversity and so I concentrated on the correlation of these two.

-Lack of proper, authentic data in the field of cultural diversity. For biological diversity I got the data from the WWF website. But for the cultural diversity there was a lot of variations. We can take the example of languages itself. Census 2001 lists just around 122 languages (rest are dialects according to the Census but that is highly contestable); the linguists in India take the total languages to be around 1635 (which is listed as the number of 'rationalised' mother tongues); People of India gives a figure of 325; and Ethnologue lists 414 languages for India. As we can see there are huge differences among different sources and none of these could be called 'unauthentic' or 'unofficial'. I have taken the data of ethnologue for the present study because I think that has an International acceptance. Moreover ethnologue clearly lists the regions in which language is spoken, which proved to be very useful for me. For other two cultural parameters - religions and ethnic groups - the scenario is even more dismal.

-Because of the time constraints. Due to the lack of a proper, organized data for the country, I had to give a lot of time for the collection and compilation of data. For example I got the animal diversity data separately by ecoregions. So I had to find them separately. And then for data on languages, I had to reconstruct the whole data, using the map of the ecoregions of India and the languages of India given in ethnologue. Comparing these two sources, I calculated the number of languages per region. Ethnologue lists the regions within which a particular language in India is spoken and ecoregion is also defined by these regions. This was very timeconsuming and the data also seems to be a bit fabricated even though I have tried to remain as close to the original data as possible. I had no other way out; so I had to do it.

Besides the calculation of this IBLD-RICH, given by Harmon and Loh, I have also calculated the Spearman's Rank Correlation Coefficient. It is a simple statistical tool to compare the ranks of two variables and see if they are correlated. It is given by the following formula:

Spearman's Rank Correlation Coefficient $(\sigma)=\left[1-\left\{\left(6 \sum d^{2}\right) / n\left(n^{2}-1\right)\right\}\right]$ Where $d=$ difference between the ranks of the two variables (for our case it is the difference between the rank for 
biological and linguistic diversity of each ecoregion) $n=$ Total number of objects for which rank is given (for our case it is the total number of ecoregions)

For the purpose of calculating this rank correlation coefficient, I have ranked the ecoregions separately according to the biological diversity (BD) score and linguistic diversity (LD) score. These two scores are calculated according to the methodology given by Harmon and Loh. Ranking is done in descending order i.e., rank 1 is given to the ecoregion that has the highest score and 37 to the one having the lowest score (total number of ecoregions being 37 in India).

All the significant calculations and findings are given in the tables included in the appendix. Table 1 gives the integrated biolinguistic diversity index; and Table 2 gives the final correlation coefficient of the ranks of biological and linguistic diversity.

\section{Analysis and Conclusion}

The biolinguistic index calculated by the method described above does not actually give the expected result. At the surface level there does not seem to be any correlation between the biological and linguistic diversity, unlike in the case of the global index calculated by Harmon and Loh (2005). However, the rank correlation coefficient does show some semblance of a correlation. The range of this coefficient is from 0 to 1 , where 0 means not correlated and 1 means completely correlated. The correlation coefficient of the ranks of biological and linguistic diversity comes out to be approx. 0.456, which is almost mid-way, although still on the lower side. It proves that they are somehow correlated but this value does not completely support the hypothesis that the two are correlated. Moreover, if we draw a bar diagram (Chart 1) to compare their index scores then we can very clearly see that there is hardly any considerable correlation between the two and there are more exceptions than rules. However with the use of some more sophisticated tools we can see that there do exist some kind of correlation. Chart 2 is a scatter diagram where we plot the value of the indices on the $\mathrm{x}$-axis and on the y-axis the ranks of the ecoregion are plotted. In the diagram that I have shown, the ranks are decided by arranging the indices of the linguistic diversity in descending order. So at the place of rank 1, we plot the value of both the biological and linguistic diversity index of the ecoregion concerned and similarly we plot the whole of the scatter diagram. Then all the scattered points of both the data are joined one by one separately. And finally a straight line is drawn through the points such that it passes through the maximum number of points. This straight line shows the 'linear regression' and it is called 'line of regression' and the rest of the points are chiefly scattered around it. Literally, 'regression' means 'stepping back towards the average'. It is a simple statistical tool which gives the measure of the average relationship between two or more variables in terms of the original unit of the data. In this case different index scores of different ecoregions are our variables and these variables are to be compared. For both biological and linguistic diversity, I have made different lines of regression on the same sheet. Now if we compare the two lines of regression we see that in both the cases the average tendency is a decrease in the index value as we move towards lower rank; so this shows the correlational pattern. However the differences are too stark to be neglected. In the case of biological diversity there is a huge amount of scattering the standard deviation from the average. Moreover from rank 1 to rank 37 there is nothing like a very significant or sharp fall in the average index score. But on the other hand in the case of linguistic diversity the scattering around the line of regression is not much (it should be so as we have arranged these indices in descending order) and there is a very considerable difference between the average score of ecoregion ranked 1 and ecoregion ranked 37.

These discrepancies in the final result can be accorded to two main reasons:

-Absence of very authentic data: The data on both plant diversity and linguistic diversity is not very reliable and their authenticity is always on the questionable side. 
-Problems in the method of calculation: Harmon and Loh themselves had pointed out that the index calculated by their method may not prove to be very useful in case of intra-country scenarios. Moreover these indices only give general conditions and they are not expected to give an in-depth analysis. And in this case, as shown in the scatter diagram by the line of regression, I think that it has indeed pointed towards the general trend which is in consonance with the hypothesis that biological and linguistic diversity are somehow correlated.

In order to make this index more powerful, efforts are now underway to develop time-series data (data calculated over a large period of time) on linguistic diversity, as well as the methodology for a locally appropriate, globally applicable indicator directly focused on trends of retention or loss of traditional environmental knowledge over time. Both of these indicators might contribute to the needs of the linguistic, cultural and biological diversity, and will help in finding an answer to one of the basic questions in the field of biocultural diversity: is the world's cultural diversity indeed in decline, and, if so, how fast? Correlated with time-series data on biodiversity, these new indicators will also show whether trends in cultural diversity and biodiversity mirror each other.

Also, an expert group on language endangerment and language maintenance gathered by UNESCO has put forth a set of recommendations for the assessment of linguistic vitality that is also expected to provide some help for the development of linguistic diversity indicators. They point out that sheer trends in "language richness" (number of different languages) are not a fully adequate indicator of the state of languages. Better data on numbers of speakers over time and other sociolinguistic "vital statistics," particularly on intergenerational language transmission, contexts of use, availability of mother tongue education, and so forth, will be needed for this purpose. A methodology has recently been developed for testing linguistic vitality at the local level and identifying the factors (such as age, gender, special roles, etc.) that affect linguistic ability.

This study of the linguistic and biological diversity within India has reaffirmed that these two (and consequently cultural diversity also) are correlated. But at the same time it has also reaffirmed the need for much better database and also some improvement in the methodology for measuring these correlations as well as the trends.

\section{References}

Carder, Maurice. 2008. A Word of Difference, from 'Resurgence Magazine', 250.

Gupta, S.C. and V.K. Kapoor. 2003. Fundamentals of Mathematical Statistics. Sultan Chand \& Sons.

Loh, Jonathan and David Harmon. 2005. A global index of biocultural diversity, from 'Ecological Indicators'.

Maffi, Luisa. 1998. Language: A Resource for Nature, from 'The UNESCO Journal on the Environment and National Resources Research', 34(4).

Maffi, Luisa. 2005. Linguistic, Cultural and Biological Diversity, from 'Annual Review of Anthropology' 2005.

Maffi, Luisa. 2007a. Biocultural Diversity and Sustainability 'The Sage Handbook of Environment and Society'.

Maffi, Luisa. 2007b. Cultural Vitality, from 'Resurgence Magazine', 250.

Skutnabb-Kangas, Tove. 2008. Linguistic Genocide in Education or Worldwide Diversity and Human Rights. Orient Longman. 


\section{Table 1}

Biolinguistic Diversity Index

\section{Eco_id Eco_code}

$\begin{array}{ll}40101 & \text { IM0101 } \\ 40105 & \text { IM0105 } \\ 40111 & \text { IM0111 } \\ 40115 & \text { IM0115 } \\ 40120 & \text { IM0120 } \\ 40124 & \text { IM0124 } \\ 40125 & \text { IM0125 } \\ 40126 & \text { IM0126 } \\ 40131 & \text { IM0131 } \\ 40133 & \text { IM0133 } \\ 40134 & \text { IM0134 } \\ 40135 & \text { IM0135 } \\ 40142 & \text { IM0142 } \\ 40150 & \text { IM0150 } \\ 40151 & \text { IM0151 } \\ 40162 & \text { IM0162 } \\ 40166 & \text { IM0166 } \\ 40201 & \text { IM0201 } \\ 40203 & \text { IM0203 } \\ 40204 & \text { IM0204 } \\ 40206 & \text { IM0206 } \\ 40207 & \text { IM0207 } \\ 40208 & \text { IM0208 } \\ 40209 & \text { IM0209 } \\ 40301 & \text { IM0301 } \\ 40303 & \text { IM0303 } \\ 40401 & \text { IM0401 } \\ 40403 & \text { IM0403 } \\ 40501 & \text { IM0501 } \\ 40502 & \text { IM0502 } \\ 40901 & \text { IM0901 } \\ 41301 & \text { IM1301 } \\ 41303 & \text { IM1303 } \\ 41304 & \text { IM1304 }\end{array}$

\section{Eco_regions}

Andaman Islands rain forests

Brahmaputra Valley semi-evergreen forests

Eastern highlands moist deciduous forests

Himalayan subtropical broadleaf forests

Lower Gangetic Plains moist deciduous forests

Malabar Coast moist forests

Maldives-Lakshadweep-Chagos

Archipelago tropical moist forests

Meghalaya subtropical forests

Mizoram-Manipur-Kachin rain forests

Nicobar Islands rain forests

North Western Ghats moist deciduous forests

North Western Ghats montane rain forests

Orissa semi-evergreen forests

South Western Ghats moist deciduous forests

South Western Ghats montane rain forests

Sundarbans freshwater swamp forests

Upper Gangetic Plains moist deciduous forests

Central Deccan Plateau dry deciduous forests

Chhota-Nagpur dry deciduous forests

East Deccan dry-evergreen forests

Khathiar-Gir dry deciduous forests

Narmada Valley dry deciduous forests

Northern dry deciduous forests

South Deccan Plateau dry deciduous forests

Himalayan subtropical pine forests

Northeast India-Myanmar pine forests

Eastern Himalayan broadleaf forests

Western Himalayan broadleaf forests

Eastern Himalayan subalpine conifer forests

Western Himalayan subalpine conifer forests

Rann of Kutch seasonal salt marsh

Deccan thorn scrub forests

Northwestern thorn scrub forests

Thar desert
Biolinguistic

Diversity Index

0.5429

0.7908

0.757

0.6254

0.8013

0.5903

NA

0.7552

0.7465

0.5478

0.6659

0.6772

0.5925

0.6535

0.6717

0.5496

0.6435

0.6416

0.7154

0.5983

0.6315

0.6817

0.6577

0.6828

0.6805

0.6263

0.7383

0.6087

0.5695

0.5682

0.5774

0.7479

0.7474

0.6155 


$\begin{array}{ll}41401 & \text { IM1401 } \\ 41406 & \text { IM1406 } \\ 81003 & \text { PA1003 } \\ 81006 & \text { PA1006 } \\ 81012 & \text { PA1012 } \\ 81021 & \text { PA1021 }\end{array}$

Godavari-Krishna mangroves

Sundarbans mangroves

NA

NA

Eastern Himalayan alpine shrub and

Karakoram-West Tibetan Plateau alpine

steppe

Northwestern Himalayan alpine shrub and

$$
\text { meadows }
$$

Western Himalayan alpine shrub and Meadows
0.50225

0.5904

0.65285

0.6074

\section{Table 2}

Calculation of Rank Correlation Coefficient

eco-regions

Andaman Islands rain forests

Brahmaputra Valley semievergreen forests

Eastern highlands moist deciduous forests

Himalayan subtropical broadleaf forests

Lower Gangetic Plains moist deciduous forests

Malabar Coast moist forests

Meghalaya subtropical forests

Mizoram-Manipur-Kachin rain forests

Nicobar Islands rain forests

North Western Ghats moist deciduous forests North Western Ghats montane rain forests

Orissa semi-evergreen forests

South Western Ghats moist deciduous forests

South Western Ghats montane rain forests

Sundarbans freshwater swamp forests

Upper Gangetic Plains moist deciduous forests Central Deccan Plateau dry deciduous forests

Chhota-Nagpur dry deciduous forests

\section{biodiversity rank}

(br)

35

6

18

14

3

12

4

1

36

9

7

26

8

5

33

11

16

24 linguistic diversity rank

(Ir)

32

1

3

25

2

33

7

8

31

17

14

30

19

18

34

22

20

6
Difference

in the rank

$d=b r-I r \quad d^{\wedge} \mathbf{2}$

9

25

225

121

1

441

9

49

25

64

49

16

121

169

1

121

16

18 
East Deccan dryevergreen forests Khathiar-Gir dry deciduous forests Narmada Valley dry deciduous forests

Northern dry deciduous forests

South Deccan Plateau dry deciduous forests

Himalayan subtropical pine forests

Northeast India-Myanmar pine forests

Eastern Himalayan broadleaf forests

Western Himalayan broadleaf forests

Eastern Himalayan subalpine conifer forests

Western Himalayan subalpine conifer forests Rann of Kutch seasonal salt marsh

Deccan thorn scrub forests

Northwestern thorn scrub forests

Thar desert

Eastern Himalayan alpine shrub and meadows

Karakoram-West Tibetan Plateau alpine steppe

Northwestern Himalayan alpine shrub and meadows Western Himalayan alpine shrub and Meadows
26

23

15

11

12

21

10

24

36

35

27

5

16

37

28

13

29

Total

10

23

$1-\left[6 \Sigma\left(d^{\wedge} 2\right) / n\left(n^{\wedge} 2-1\right)\right]$

0.456140351

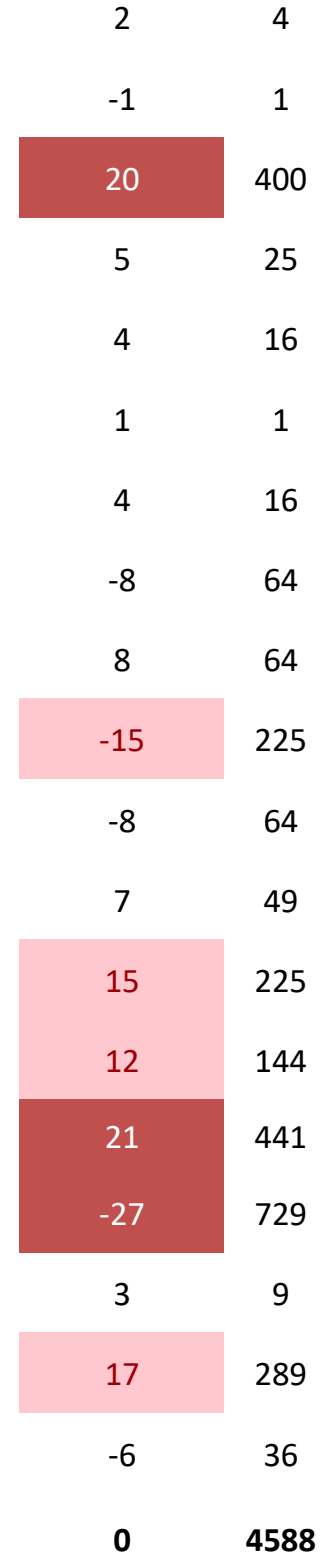

$27.03 \%$

$10.81 \%$

$62.16 \%$
25

$\begin{array}{cc}\text { BADCORRELATION } & 10 \\ \text { V.BADCORRELATION } & 4 \\ \text { SOMEWHAT } & 23 \\ \text { CORRELATED } & \\ \text { Rank Correlation } & 1-\left[6 \Sigma\left(\mathrm{d}^{\wedge} 2\right) / \mathrm{n}\left(\mathrm{n}^{\wedge} 2-1\right)\right] \\ \text { Coefficient }= & 0.456140351\end{array}$




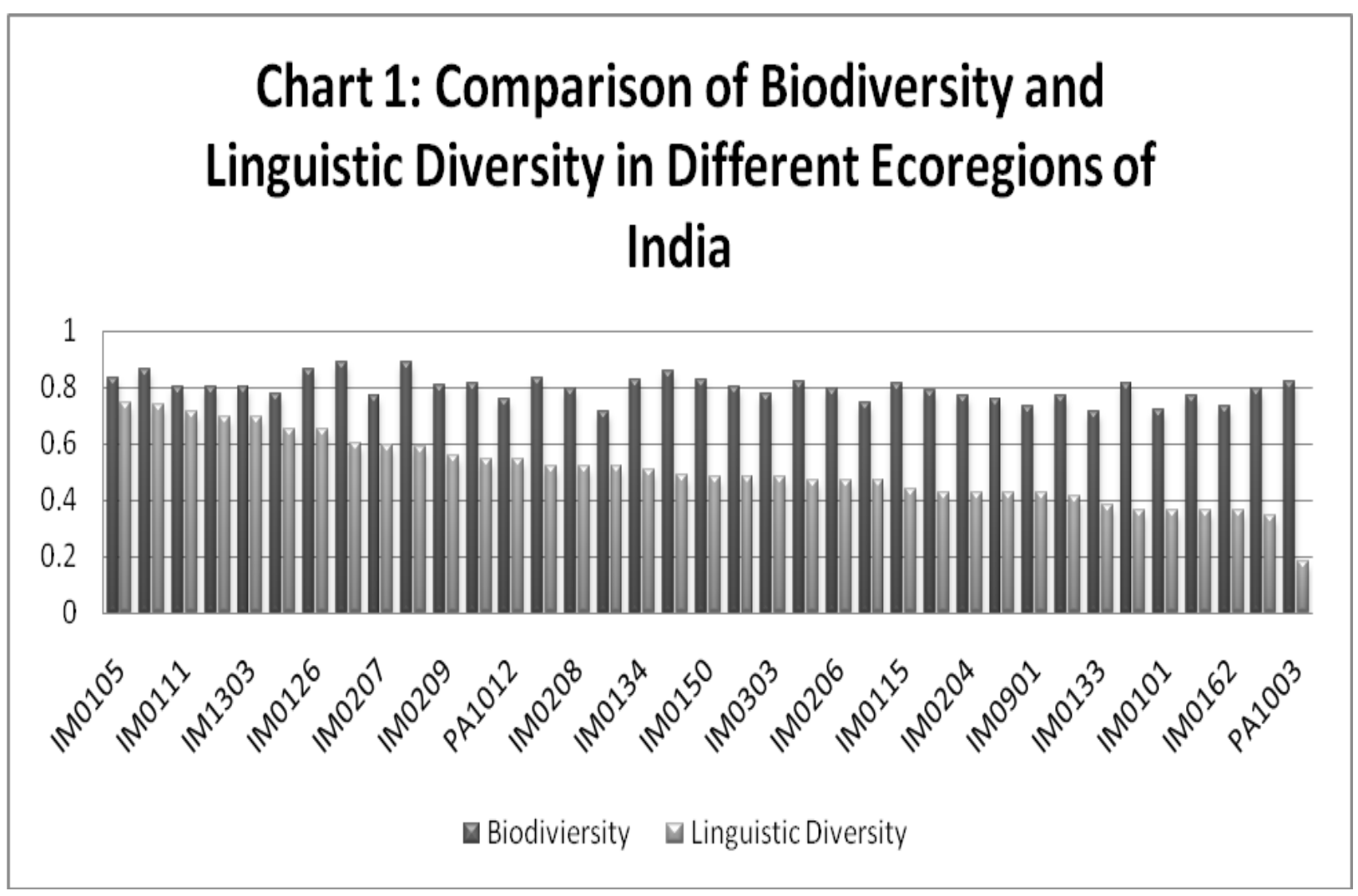

